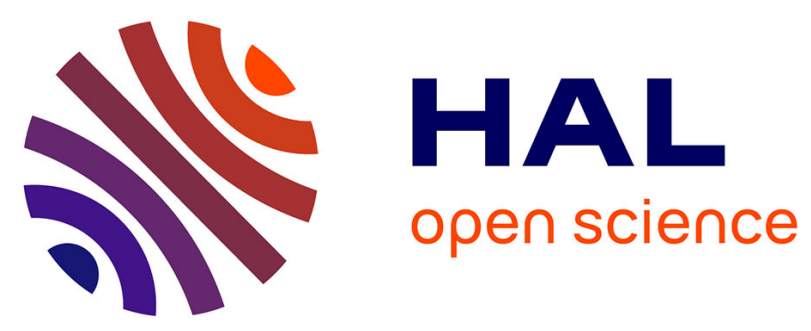

\title{
Refinements on electron diffraction data of $\beta$-glycine in MoPro: a quest for an improved structure model
}

\author{
Kunal Kumar Jha, Barbara Gruza, Michal Leszek Chodkiewicz, Christian
}

Jelsch, Paulina Maria Dominiak

\section{- To cite this version:}

Kunal Kumar Jha, Barbara Gruza, Michal Leszek Chodkiewicz, Christian Jelsch, Paulina Maria Dominiak. Refinements on electron diffraction data of $\beta$-glycine in MoPro: a quest for an improved structure model. Journal of Applied Crystallography, 2021, 54 (4), pp.1234 - 1243. $10.1107 / \mathrm{s} 160057672100580 \mathrm{x}$. hal-03313174

\section{HAL Id: hal-03313174 \\ https://hal.science/hal-03313174}

Submitted on 3 Aug 2021

HAL is a multi-disciplinary open access archive for the deposit and dissemination of scientific research documents, whether they are published or not. The documents may come from teaching and research institutions in France or abroad, or from public or private research centers.
L'archive ouverte pluridisciplinaire HAL, est destinée au dépôt et à la diffusion de documents scientifiques de niveau recherche, publiés ou non, émanant des établissements d'enseignement et de recherche français ou étrangers, des laboratoires publics ou privés. 


\section{Refinements on electron diffraction data of $\beta$-glycine in MoPro: a quest for an improved structure model}

\section{Kunal Kumar Jha, Barbara Gruza, Michał Leszek Chodkiewicz, Christian Jelsch and Paulina Maria Dominiak}

J. Appl. Cryst. (2021). 54, 1234-1243

\section{IUCr Journals CRYSTALLOGRAPHY JOURNALS ONLINE}

Author(s) of this article may load this reprint on their own web site or institutional repository provided that this cover page is retained. Republication of this article or its storage in electronic databases other than as specified above is not permitted without prior permission in writing from the IUCr.

For further information see https://journals.iucr.org/services/authorrights.html 


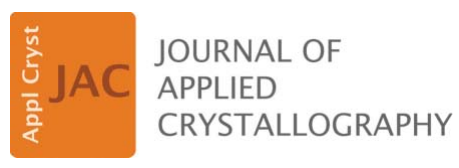

ISSN 1600-5767

Received 13 December 2020

Accepted 3 June 2021

Edited by S. Boutet, SLAC National Accelerator Laboratory, Menlo Park, USA

Keywords: electron diffraction; multipolar atoms database; independent atom model (IAM); transferred aspherical atom model (TAAM); MoPro; quantum crystallography; structure refinement.

CCDC references: 2087927; 2087928; 2087929; 2087930; 2087931

Supporting information: this article has supporting information at journals.iucr.org/j

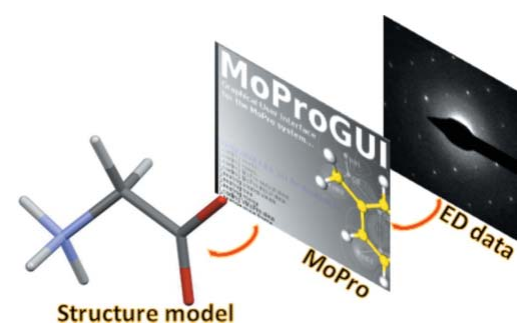

\section{Refinements on electron diffraction data of $\beta$-glycine in MoPro: a quest for an improved structure model}

\author{
Kunal Kumar Jha, ${ }^{a}$ Barbara Gruza, ${ }^{a}$ Michał Leszek Chodkiewicz, ${ }^{a}$ Christian Jelsch ${ }^{\text {b* }}$ \\ and Paulina Maria Dominiak ${ }^{a}$.
}

aBiological and Chemical Research Centre, Department of Chemistry, University of Warsaw, ulica Żwirki i Wigury 101, 02-089, Warszawa, Poland, and ${ }^{\mathbf{b}}$ Université de Lorraine, CNRS, CRM2, Nancy, France. *Correspondence e-mail: christian.jelsch@univ-lorraine.fr, pdomin@chem.uw.edu.pl

The advancement in 3D electron diffraction (3D ED) techniques that lead to a revolution in molecular structure determination using nano-sized crystals is now achieving atomic resolution. The structures can be obtained from 3D ED data with tools similar to those used for X-ray structure determination. In this context, the MoPro software, originally designed for structure and charge density refinements using X-ray diffraction data, has been adapted. Structure refinement on 3D ED data was achieved via implementation of electron scattering factors available in the literature and by application of the MottBethe equation to X-ray scattering factors computed from the multipolar atom model. The multipolar model was parametrized using the transferable pseudoatom databanks ELMAM2 and UBDB. Applying the independent atom model (IAM), i.e. spherical neutral atom refinement, to 3D ED data on $\beta$-glycine in MoPro resulted in structure and refinement statistics comparable to those obtained from other well known software. Use of the transferred aspherical atom model (TAAM) led to improvement of the refinement statistics and a better fit of the model to the 3D ED data as compared with the spherical atom refinement. The anisotropic displacement parameters of non- $\mathrm{H}$ atoms appear underestimated by typically $0.003 \AA^{2}$ for the non- $\mathrm{H}$ atoms in IAM refinement compared with TAAM. Thus, MoPro is shown to be an effective tool for crystal structure refinement on 3D ED data and allows use of a spherical or a multipolar atom model. Electron density databases can be readily transferred with no further modification needed when the Mott-Bethe equation is applied.

\section{Introduction}

The most conclusive and elucidating component of any smallmolecule or macromolecular study is a definitive structure determination. The complex nature of drug and target protein interactions can only be studied effectively by determining their 3D structures. The two most commonly used tools for structure determination are NMR spectroscopy and X-ray diffraction. While both these techniques are extremely popular, they have certain limitations. A new technique, electron cryo-microscopy (cryo-EM), has taken a leap in the past few years. The cryo-EM method known as 3D electron diffraction (3D ED) has also been reported to provide atomic resolution structures of various small molecules, pharmaceutically active mixtures, zeolites, metal organic frameworks and macromolecules (Nannenga et al., 2014; Yonekura et al., 2015; van Genderen et al., 2016; Gemmi et al., 2019; Gruene et al., 2018; Jones et al., 2018; Mugnaioli et al., 2020; Beale et al., 2020). 3D ED data are collected by rotating the crystal inside a transmission electron microscope. Unlike X-ray diffraction, 
where the X-ray beam is scattered by the electron density, in 3D ED, the electron beam is scattered by the electrostatic (Coulomb) potential of the sample. Apart from advances in technology for electron diffraction instruments (Kolb et al., 2011; Wang et al., 2019; Hattne et al., 2019; Gemmi et al., 2019; Polovinkin et al., 2020), the major reason for drawing the attention of chemists and biologists to 3D ED is the nano-size of crystals. With 3D ED it is possible to obtain high-resolution data from nano-crystals with an accuracy approaching that of $\mathrm{X}$-ray diffraction data and to determine the absolute configuration (Sawaya et al., 2016; Jones et al., 2018; Gruene et al., 2018; Krysiak et al., 2018; Clabbers et al., 2019; Brázda et al., 2019; Xu \& Zou, 2019), something that is impossible with $\mathrm{X}$-ray diffraction.

The advantage of electron diffraction over X-ray diffraction is that it is more sensitive to valence charge densities than $\mathrm{X}$-ray diffraction in the $0.2-0.6 \AA^{-1}$ resolution range (Mott \& Massey, 1965; Zheng et al., 2005). At this low resolution, the effect of atomic thermal displacement is relatively small compared with that in the higher-resolution range, and structural information related to chemical bonding properties can be obtained (Zuo et al., 1999; Nakashima, 2017). The disadvantage of electron diffraction is the presence of dynamic scattering effects, which need to be taken into account properly and require large computational effort (Nakashima et al., 2011; Palatinus et al., 2015; Nakashima, 2017) or some approximate statistical corrections (Clabbers et al., 2019). It has been shown, however, that the use of relatively thin crystals together with crystal rotation or beam precession helps to substantially limit multiple scattering and allow for structure solution and refinement with minimal impact on the quality of the structure model thus obtained (Cowley \& Moodie, 1957; Clabbers et al., 2017; Nannenga et al., 2014; Nannenga \& Gonen, 2019; Gemmi et al., 2019; Blum et al., 2021).

The electrostatic potential (ESP) obtained via scattering of electrons hitting the sample is considered equivalent to the scattering potential (Peng, 1999). Once the intensities are extracted, the structures are obtained from the 3D ED data using tools similar to those used for X-ray diffraction structure determination, like SHELX (Sheldrick, 2015), OLEX2 (Dolomanov et al., 2009) etc. Most often, the independent atom model (IAM) is used for solving and refining the structures (Petříček et al., 2014; Brown et al., 2015). IAM is a model of ESP built using scattering factors precomputed for isolated, spherically averaged atoms or ions. It was shown, however, that the local electric charges and ionization states significantly affect the electron scattering factors (Yonekura et al., 2015; Yonekura \& Maki-Yonekura, 2016). The use of improper electron scattering factors in refinement may lead to physically unrealistic atomic positions and unacceptable refinement statistical values (Shi et al., 2013; Nannenga et al., 2014; de la Cruz et al., 2017). To overcome this, it was proposed that partially charged electron scattering factors be used for refinement, which improved the fitting statistics and other physical properties (Yonekura \& Maki-Yonekura, 2016). In reality, the atoms in molecules are neither isolated nor sphe- rical. Use of an aspherical model should lead to an improved description of atomic positions (Zhong et al., 2002; Zheng et al., 2009).

With the knowledge that atoms in similar chemical environments have similar charge densities (Brock et al., 1991), a number of pseudoatom databases have been created based on the Hansen \& Coppens (1978) multipolar atom formalism. Multipolar database transfer enables one to improve the X-ray structure model and speed up the charge density study of small and macromolecular structures (Zarychta et al., 2007; Domagała et al., 2012; Nassour et al., 2017; Dittrich et al., 2004, 2013; Volkov et al., 2007; Dominiak et al., 2007; Jarzembska \& Dominiak, 2012; Kumar et al., 2019). A multipolar approach using the transferable aspherical atom model (TAAM) refinement applied for X-ray structure refinement results in a substantially improved physical representation of crystals, including atomic positions and anisotropic atomic displacement parameters (ADPs) (Jelsch et al., 1998; Jha et al., 2020). Better descriptions of $\mathrm{H}$-atom positions and accurate hydrogen bond lengths, comparable to reference neutron bond lengths, with improved refinement statistics are achieved using TAAM with the UBDB (Jha et al., 2020) or ELMAM2 (Zarychta et al., 2007) databanks on X-ray diffraction data.

Similar improvements in statistics and atomic positions were also achieved in electron TAAM UBDB refinement of simulated 3D ED data of carbamazepine (Gruza et al., 2020). Improvements of TAAM UBDB refinement with experimental 3D ED data were less satisfactory, probably because the refinements were not corrected for dynamic scattering effects.

Here we present the results of spherical IAM and aspherical TAAM UBDB and TAAM ELMAM2 refinements with 3D ED data for another small-molecule crystal, $\beta$-glycine (Fig. 1 and Table 1), for which the 3D ED data were already available (Broadhurst et al., 2020). Glycine is the simplest amino acid. There are six known polymorphs of glycine and all the polymorphs exist in zwitterionic form. $\beta$-Glycine is the least stable polymorph under ambient conditions and exists in a non-centrosymmetric monoclinic form (space group $P 2_{1}, Z=$ 2) (Perlovich et al., 2001; Boldyreva et al., 2003). The refinements were performed with the MoPro software. MoPro is a

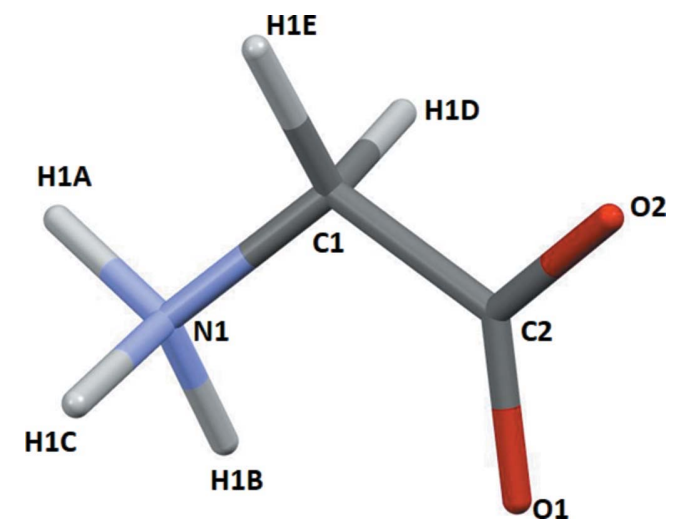

Figure 1

$\beta$-Glycine structure with atomic numbering scheme. The same atomic numbering scheme was used in further analysis. 
Table 1

Crystallographic data.

\begin{tabular}{ll}
\hline Formula & $\mathrm{C}_{2} \mathrm{H}_{5} \mathrm{NO}_{2}$ \\
Formula weight & 75.07 \\
Space group & $P 2_{1}$ \\
$a(\AA)$ & $5.3110(11)$ \\
$b(\AA)$ & $6.4540(13)$ \\
$c(\AA)$ & $5.6940(11)$ \\
$\beta\left(\left(^{\circ}\right)\right.$ & $112.86(3)$ \\
Volume $\left(\AA^{3}\right)$ & $179.84(7)$ \\
$Z ; Z^{\prime}$ & $2 ; 1$ \\
Density $\left(\mathrm{g} \mathrm{cm}^{-3}\right)$ & 1.387 \\
No. unique reflections & 859 \\
No. reflections with $I>2 \sigma$ & 736 \\
Maximal resolution $d(\AA) / \sin \theta / \lambda\left(\AA^{-1}\right)$ & $0.75 / 0.67$ \\
\hline
\end{tabular}

structure and charge density refinement software utilized in the past for various studies including small organic and macromolecular protein systems (Guillot et al., 2001; Jelsch et al., 2005, 2020; Guillot et al., 2008; Bouhmaida et al., 2009; Niranjana Devi et al., 2017). Aspherical TAAM refinement using ELMAM2 database transfer on X-ray experimental data in MoPro is a very convenient and user-friendly tool (Zarychta et al., 2007; Domagała et al., 2012; Bibila Mayaya Bisseyou et al., 2012). ELMAM2 (Domagała et al., 2012) is a generalized and improved library of transferable, experimentally derived multipolar data. For the purpose of this study, electron scattering factors within the IAM and TAAM approximations were implemented in MoPro. Aspherical electron TAAMs were constructed using the ELMAM2 and UBDB databanks. Statistical comparison of refinement parameters and atomic positions has been done for IAM refinement in SHELX (Sheldrick, 2015) and spherical IAM and aspherical TAAM refinements using MoPro. Thereby, the MoPro program is established as a tool for spherical and aspherical refinement of 3D ED data in small molecules.

The MoPro software can be obtained by registering at https://crm2.univ-lorraine.fr/lab/fr/software/mopro/.

\section{Methodology}

\subsection{Implementation of electron scattering factors in MoPro}

The refinement against electron diffraction was implemented in two ways in the MoPro software. The first method is using IAM scattering factors computed directly for electron diffraction. These are, for example, represented as a sum of five Gaussian functions for each chemical element as a function of reciprocal resolution $s=\sin \theta / \lambda$ in International Tables for Crystallography (Prince, 2006, Tables 4.3.2.2 and 4.3.2.3). Alternatively, Peng (1999) published the scattering factors as a function of four Gaussian functions. The format of these files is given in the supporting information (Tables S1 and S2).

In the second method, the Mott-Bethe formula was used. This formula is derived from the fact that the electron density is proportional to the Laplacian of the electrostatic potential. As a result, in the Fourier space, the electron scattering factors $f^{\mathrm{e}}(\mathbf{h})$ are related to the X-ray scattering factors $f^{\mathrm{x}}(\mathbf{h})$ by (Peng, 1999)

$$
f^{\mathrm{e}}(\mathbf{h})=\frac{m_{0} e^{2}}{8 \pi^{3} \hbar^{2} \varepsilon_{0}} \frac{Z-f^{\mathrm{x}}(\mathbf{h})}{\mathbf{h}^{2}},
$$

where $|\mathbf{h}|=2 s=2 \sin \theta / \lambda$ is the reciprocal resolution, $m_{0}$ and $e$ are the rest mass and charge of the electron, $h$ equals $h / 2 \pi$ where $h$ is the usual Planck constant, $\varepsilon_{0}$ is the vacuum permittivity, and $Z$ is the atomic number of the atom. To obtain $f^{\mathrm{e}}(\mathbf{h})$ in ångström units from $f^{\mathrm{x}}(\mathbf{h})$ in electron units the following multiplier was used:

$$
f^{\mathrm{e}}(\mathbf{h})=0.095736 \frac{Z-f^{\mathrm{x}}(\mathbf{h})}{\mathbf{h}^{2}} .
$$

Note that the multiplier is four times larger than the original value of 0.023934 given by Peng (1999). This is to account for the fact that reciprocal resolution is represented by $|\mathbf{h}|$ and not $s=\frac{1}{2}|\mathbf{h}|$.

X-ray scattering factors are computed from the Hansen \& Coppens (1978) multipolar model parametrized with the use of the ELMAM2 (Domagała et al., 2012) or UBDB2018 (Kumar et al., 2019; Volkov et al., 2004) databank.

Different scattering factors $f^{\mathrm{e}}(s)$ as a function of reciprocal resolution are shown in Fig. 2. The graphs show the great variety of $f^{\mathrm{e}}(s)$ curves, which depend on the atomic charge. The IAM carbon atom and a positively charged $(+1 \mathrm{e})$ carbon atom have $f^{\mathrm{e}}(s)$ positive and monotonically decreasing to zero at higher resolution. However, the scattering of the IAM carbon atom is much more significant at high resolution than that of a charged one. This is because the ESP of an IAM atom decreases to zero with distance $r$ much more rapidly than for

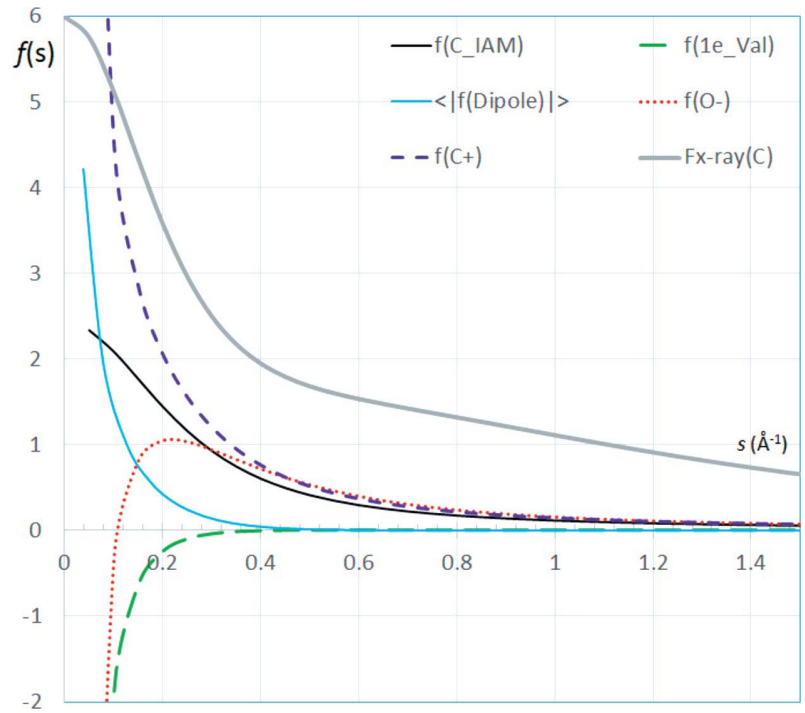

Figure 2

Electron scattering factors $(\AA)$ as a function of reciprocal resolution $s=$ $\sin \theta / \lambda\left(\AA^{-1}\right)$ for an IAM carbon atom, f(C_IAM), a carbon atom with charge $+1 \mathrm{e}, \mathrm{f}(\mathrm{C}+)$, an oxygen atom with charge $-1 \mathrm{e}, \mathrm{f}(\mathrm{O}-)$, and a population of one electron from the IAM carbon valence shell, $\mathrm{f}(1 \mathrm{e}$ - Val $)$. The spherically averaged absolute value of $f^{\mathrm{e}}(s)$ for a dipole with population $P_{10}=1\left(\kappa^{\prime}=1\right)$ on a carbon atom is also shown, \langle| $\mathrm{f}($ Dipole $)|\rangle$. The $f^{\mathrm{e}}(s)$ values were computed with the MoPro program using the MottBethe formula [equations (1) and (2)]. For comparison, the X-ray scattering factor (e) of an IAM carbon atom is added, Fx-ray(C). 
an atom of charge $q$, for which the ESP at long distance shows a very slow decrease in $q / r$.

An oxygen atom with charge -1 e has conversely an $f^{\mathrm{e}}(s)$ value which is negative at very low resolution $\left(s<0.1 \AA^{-1}\right)$ and becomes positive at higher resolution. Charged atoms have $f^{\mathrm{e}}(s)$ tending towards $\pm \infty$ when $s \rightarrow 0$, depending on the sign of the charge, while for a neutral IAM atom, $f^{\mathrm{e}}(s)$ tends to a finite number. The electron scattering factors of a carbon valence electron and a dipole have $f^{\mathrm{e}}(s)$ scattering tending towards zero much more rapidly with $s$, as observed also by X-ray diffraction.

The scattering factors obtained from the present study for a carbon atom with charge $+1 \mathrm{e}, f\left(\mathrm{C}^{+}\right)$, and an oxygen atom with charge -1 e, $f\left(\mathrm{O}^{-}\right)$, are similar to results reported previously by Yonekura et al. (2018) [Figs. S1 $(a)$ and S1 $(b)$ ]. They differ by the $R$ factors $\mathrm{Rf}=20.0$ and $16.2 \%$ for $f\left(\mathrm{C}^{+}\right)$and $f\left(\mathrm{O}^{-}\right)$, respectively. The reported $f^{\mathrm{e}}(s)$ values were derived from the wavefunctions computed directly for isolated ions. In the current study, the $f^{\mathrm{e}}(s)$ values are derived from isolated neutral atom wavefunctions (Clementi \& Roetti, 1974) for which the X-ray scattering factors for core and valence electrons are considered separately. The scattering factors of the ionic atoms in Fig. 2 were computed with the valence electron scattering factors rescaled to the appropriate $P_{\text {val }}$ number of valence electrons: $f=f_{\text {core }}+P_{\text {val }} f_{\text {val }}$. The $f\left(\mathrm{O}^{-}\right)$value of the oxygen ion calculated directly from the isolated $\mathrm{O}^{-}$ion wavefunction following the same quantum mechanical methodology (Clementi \& Roetti, 1974) is very similar to the $f\left(\mathrm{O}^{-}\right)$ value reported by Yonekura et al. (2018), with only $0.07 \%$ difference [Fig. S1(b)]. A major difference between the $f(s)$ value computed on the isolated $\mathrm{O}^{-}$ion and the $f=f_{\text {core }}+$ $P_{\text {val }} f_{\text {val }}$ approach is that the expansion of the $\mathrm{O}^{-}$electron density is neglected $(\kappa=1.0)$ in the latter case and causes a discrepancy with the $f\left(\mathrm{O}^{-}\right)$value of Yonekura et al. (2018) [Figs. S1 $(a)$ and $\mathrm{S} 1(b)$ ]. In the TAAM refinement of the glycine structure, the scattering factors used for the multipolar charged atoms are more sophisticated as they take into account, in addition to $P_{\mathrm{val}}$, the $\kappa$ expansion/contraction parameter of the valence electron density [Fig. S1 $(c)$ ]. This is a common approach in pseudoatom modelling of organic molecules, since partial charges on atoms in organic molecules rarely exceed values of $\pm 0.5 \mathrm{e}$.

\subsection{Madelung potential and $\boldsymbol{F}_{000}$ value}

The $F_{000}$ term (structure factor of the $h=0, k=0, l=0$ reflection) is not necessary and not used at all in the crystallographic refinement or for the computation of the residual Fourier maps. However, to compute the total periodic potential, the term $F_{000}$ is required. Also, the average electrostatic potential $\Phi$ in the crystal unit cell (the Madelung potential) is related to the $F_{000}$ value.

According to International Tables for Crystallography (Prince, 2006), the following relation exists:

$$
\Phi=47.8780 F_{000} / V_{\mathrm{uc}}
$$

when $\Phi$, the ESP, is expressed in volts, $F_{000}$ in ångström and $V_{\text {uc }}$, the unit-cell volume, in $\AA^{3}$.

Equation (1) exhibits a singularity at $|\mathbf{h}|=0$ when one attempts to compute the $F_{000}$ term. $F_{000}$ corresponds to the Madelung potential or average potential in the crystal. Becker \& Coppens (1990) have proposed methods to compute the Coulomb potential in crystals for charged multipolar atoms.

For IAM atoms, the average potential can be computed easily in direct space. For neutral spherical atoms, the ESP is positive and rapidly decreases to zero with distance [Fig. S2(a)]. A way to obtain the average ESP is then to integrate the potential $\Phi_{\mathrm{a}}$ generated by each atom of the asymmetric unit:

$$
\langle\Phi\rangle=\sum_{\text {atoms }} \iiint \Phi_{\mathrm{a}}(\mathbf{r}) \mathrm{d}^{3} \mathbf{r} / V_{\mathrm{au}}
$$

where $V_{\text {au }}$ is the volume of the asymmetric unit.

$I_{\mathrm{a}}$, the cubature of $\Phi_{\mathrm{a}}$ over space, can be obtained in spherical coordinates over a typical interval $r=0.01-5 \AA$ (Fig. S2) around atoms and can be tabulated for all chemical species:

$$
I_{\mathrm{a}}=4 \pi \int \Phi_{\mathrm{a}}(r) r^{2} \mathrm{~d} r
$$

The average IAM potential is then obtained by a linear equation using the chemical content of the asymmetric unit:

$$
\langle\Phi\rangle=\left(\sum_{\mathrm{atoms}} I_{\mathrm{a}}\right) / V_{\mathrm{au}} \text {. }
$$

The $I_{\mathrm{a}}$ values obtained for $\mathrm{H}, \mathrm{C}, \mathrm{N}$ and $\mathrm{O}$ atoms are, respec-

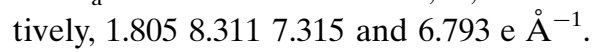

For $\beta$-glycine, the IAM Madelung potential was found to be $\langle\Phi\rangle=0.518$ e $\AA^{-1}=7.45 \mathrm{~V}$.

As $F_{000}=\langle\Phi\rangle V_{\mathrm{uc}} / 47.8780$, where the ESP is expressed in volts and the unit-cell volume $V_{\mathrm{uc}}=179.8 \AA^{3}$ (Prince, 2006), the resulting $F_{000}=28.0 \AA$

The $F_{000}$ IAM value can, on the other hand, be computed (by taking $s=0 \AA^{-1}$ ) when using the sum of Gaussian electron scattering factors as described by Peng (1999) or in International Tables for Crystallography (Prince, 2006). This $F_{000}$ value corresponding to the IAM model is written in the output Fourier file by MoPro. For the $\beta$-glycine crystal, both methods yield $F_{000}=27.6 \AA$, a value close to that obtained by real-space integration of the IAM ESP.

\subsection{Refinement criteria and procedures}

The electron diffraction data and starting structural model for $\beta$-glycine were taken from the article by Broadhurst et al. (2020). The diffraction data were first merged in MoPro before the structure refinement. The statistical weighting scheme $(w)$ based on uncertainties $(\sigma)$ associated with each reflection intensity $\left(I_{\text {obs }}\right)$ was employed in the refinement using MoPro, as shown in equation (7):

$$
w=1 / \sigma^{2}\left(I_{\mathrm{obs}}\right) \text {. }
$$

The advantage of using this simple statistical weighting scheme is that a reasonably good quality of structure model can be obtained with convergence, without suppressing the experimental and model errors (Watkin, 2008). To establish 
one-to-one comparison of refinement statistics between IAM in SHELX and MoPro, the SHELX refinements were also performed with the same statistical weight on merged data. Initial evaluation of the reported data showed smearing in the displacement ellipsoids with flat and oblate shapes, especially in the carboxylate group, indicating a systematic error in the diffraction data [Fig. S3( $a)$ ] with poor respect of the rigid bond (Thorn et al., 2012). Restraints on $U^{i j}$ such as rigid-bond restraints RIGU, SIMU and ISOR improved the displacement ellipsoids in the SHELX refinement [Fig. S3(b)]. Similar restraints were used in spherical IAM and aspherical TAAM refinement in MoPro [Figs. S4(a)-S4(b)].

All the refinements were performed against $|F(\mathbf{H})|^{2}$. In all the refinements using MoPro, the following steps were applied: $(a)$ scale factor refinement; $(b)$ isotropic refinement of non- $\mathrm{H}$ and $\mathrm{H}$ atoms; $(c)$ anisotropic refinement of non- $\mathrm{H}$ and isotropic refinement of $\mathrm{H}$ atoms. In the refinement, all the reflections after the data merging (859) were used (Table 1), and a default reflection threshold of $I>2 \sigma(I)$ was used to compute $R$-factor statistics. In the case of IAM in SHELX, electron scattering factors reported by Peng (1999) were used to model the structure. In MoPro, the IAM refinement was performed using electron scattering factors reported by Peng (1999) or taken from International Tables for Crystallography (IT) (Prince, 2006) to model the structure. In the case of TAAM refinement in MoPro, after scale refinement, additionally the ELMAM2 or UBDB databank transfer step was added. ELMAM2 database transfer is already built into MoPro. UBDB database transfer was performed externally using the LSDB code (Volkov et al., 2004) and the UBDB databank (Kumar et al., 2019).

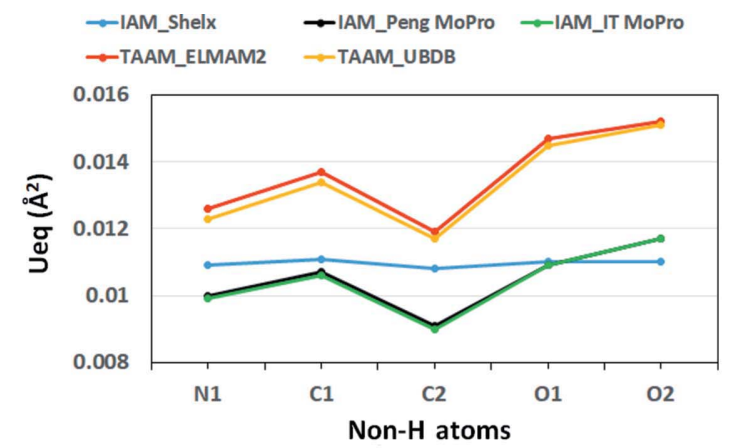

(a)

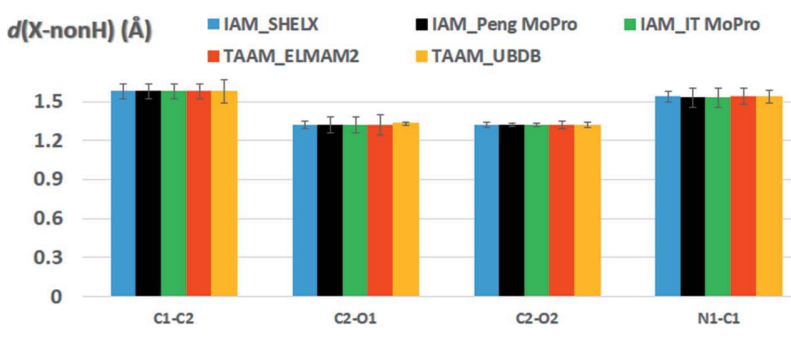

(c)

\subsection{ESP map generation in the MoPro viewer}

The ESP maps on a single molecule were generated with $V M o$ Pro using the parameter file after final refinement in VMoPro. In the 'Map Properties' tab, 'Electrostatic Potential' is selected and for $(a)$ total ESP 'The Total Electron Density (Nuclei, Core, Valence Population (Pval), all multipoles $(\mathrm{Plm}))^{\prime}$ is selected, while for $(b)$ deformation ESP 'Deformation (Pval, Kappa, Plm - Nval, Kappa $=1, \mathrm{Plm}=0$ )' is selected. In the 'Plane' parameters tab, a suitable plane and plane options are selected and 'Run VMopro' is clicked.

To obtain the quasi-periodic ESP maps, contributions from molecules generated by $19^{3}=6859$ unit cells obtained by translations up to $[-9,9](\mathbf{a}, \mathbf{b}, \mathbf{c})$ unit-cell vectors were taken into account.

\section{Results and discussion}

\subsection{IAM refinement in SHELX and MoPro}

IAM refinement in SHELX and MoPro resulted in very similar statistics. The refinement statistics such as reliability factor (Rf) and residual ESP were comparable for the two programs (Table 2). Similar minimal and maximal peaks were observed in the Fourier residual map after MoPro IAM refinement compared with $S H E L X$. The equivalent isotropic displacement parameters $\left(U_{\mathrm{eq}} / U_{\text {iso }}\right)$, which represent anisotropic and isotropic atomic displacement parameters (ADPs), for non- $\mathrm{H}$ and $\mathrm{H}$ atoms, respectively, were found to be very similar [Figs. 3(a) and 3(b)]. The bond lengths for non-H and $\mathrm{H}$ atoms were also found to be the same in both the refinements [Table S3, and Figs. 3(c) and 3(d)].

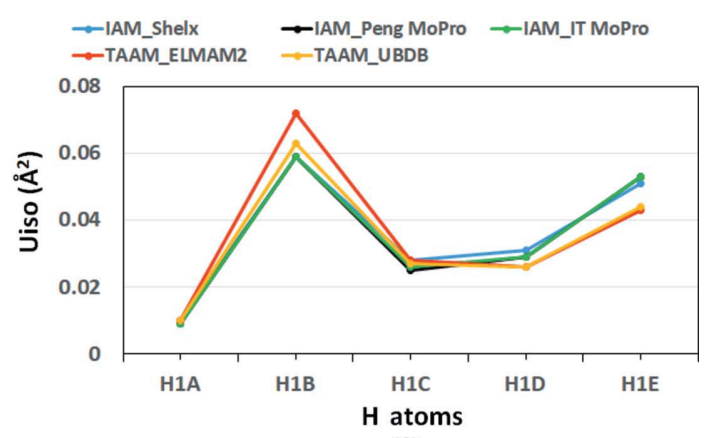

(b)

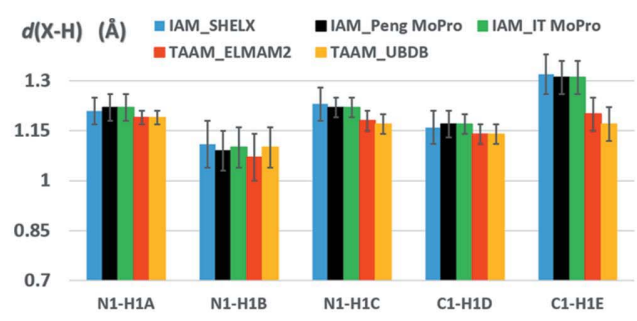

(d)

Figure 3

Comparison of IAM (SHELX and MoPro) refinements and TAAM (MoPro) refinements. Values of $U_{\text {eq }}$ and $U_{\text {iso }}$ for non-H $(a)$ and H atoms $(b)$. Bond lengths for non- $\mathrm{H}(c)$ and $\mathrm{H}$ atoms $(d)$ for IAM and TAAM refinements. 


\subsection{TAAM refinement in MoPro}

It was proved for X-ray diffraction that, although the methodologies of generation of different pseudoatom databases are different, the end results of TAAM refinements are very similar (Back et al., 2011). TAAM refinements with 3D ED data in MoPro were performed using the ELMAM2 and UBDB databanks to compare the refinements using different pseudoatom databases. While the ELMAM2 databank is based on experimental multipolar parameters, the UBDB databank is derived from theoretical charge density distributions by fitting the pseudoatom parameters to the diffraction patterns of a large number of small molecules (Kumar et al., 2019). A comparison between TAAM refinement using the ELMAM2 and UBDB databanks showed very similar refinement statistics such as Rf and residuals (Table 2). The $U_{\text {eq }}$ parameters for non-H atom and $U_{\text {iso }}$ for $\mathrm{H}$ atoms were also very similar in the two refinements [Figs. $3(a)$ and $3(b)]$. $U_{\text {iso }}$ for $\mathrm{H} 1 B$ in the ELMAM2 refinement was found to be unusually high [Fig. 3(b)]. The bond lengths for non-H atoms were very similar [Fig. 3(c)], but some differences in hydrogen $\mathrm{H}-X$ bond lengths were observed between the ELMAM2 and UBDB refinements [Fig. 3(d) and Table S3].

\subsection{Differences between IAM and TAAM refinement in MoPro}

In comparison with IAM refinement, TAAM showed improvement in the overall refinement statistics. The $\mathrm{Rf}$ and Fourier residuals both decrease slightly (Table 2 and Fig. 4). This observation is consistent with the TAAM refinement of carbamazepine by Gruza et al. (2020), where a decrease by approximately $1-2 \%$ in $\mathrm{Rf}$ was observed. This indicates better fitting to diffraction data of the more realistic multipolar model TAAM compared with IAM. More advanced analysis of the Fourier residual ESP of the entire unit cell, based on a fractal dimension plot, further confirms that TAAM refinement leads to a better fit. The parabolic shape of the fractal dimension plot is an indicator of Gaussian noise distribution on a residual map, and data are considered devoid of any systematic error (Meindl \& Henn, 2008). The fractal dimensions of the two IAM refinements (Peng and IT) were very similar and found to be overlapping with each other. The fractal dimensions of the TAAM refinements (ELMAMA2 and UBDB) were also very similar, but there were slight differences (Fig. 5). The TAAM refinement showed some improvement in fractal dimension with less broadness of the curve and evenly distributed residuals with lower $\Delta \Phi_{\mathrm{o}}$ compared with IAM (Fig. 5). Overall the Rf values from TAAM were found to be lower than those from IAM. The comparison of $\mathrm{Rf}$ at different resolutions indicated that TAAM fits better to low-resolution regions of 3D ED data (up to $\sin \theta / \lambda=0.5 \mathrm{~A}^{-1}$ ) than IAM. In the high-resolution region, the trend is not clear with the TAAM Rf either higher or lower than the IAM Rf values (Fig. 6). This effect may be due to the dynamic scattering which, on average, augments the diffraction at high resolution versus low resolution (Clabbers et al., 2019).

There is a slight reduction of the Fourier residual ESP (Table 2 and Fig. 4); an improvement of $c a 0.1 \mathrm{e} \AA^{-1}$ (= $0.03 \AA^{-2}$ ) in Fourier residual maps was found for carbamazepine (Gruza et al., 2020). In relative values, the lowering of Rf and residual ESP $\Delta \Phi$ are modest owing to the high $\mathrm{Rf}$ of around $13 \%$ of the $3 \mathrm{D}$ ED data. For X-ray crystal structures,

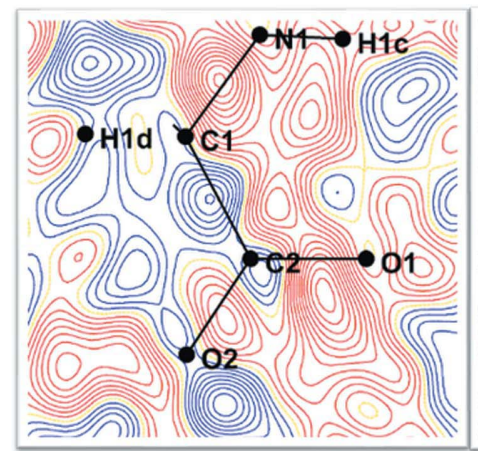

IAM_Peng

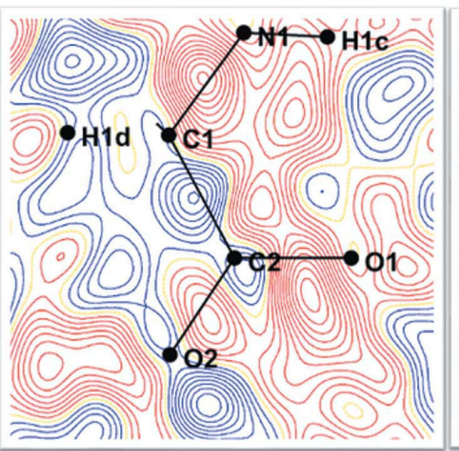

IAM_IT

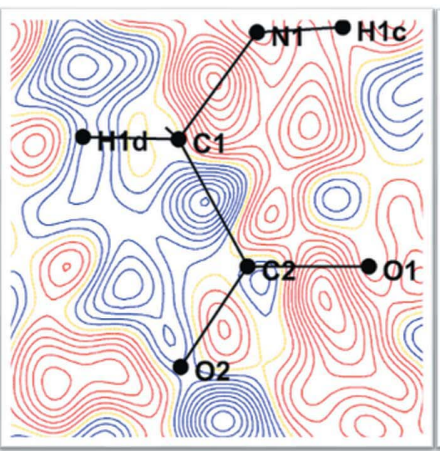

TAAM_ELMAM2

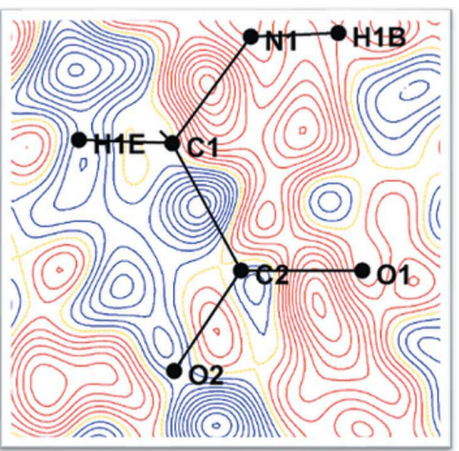

TAAM_UBDB

Figure 4

Comparison of Fourier residual maps for IAM (MoPro) and TAAM refinements. Contours are at $0.05 \AA^{-2}$ intervals (blue - positive and red - negative). 
the reduction in Rf obtained by TAAM refinement amounts to typically ca $1-2 \%$, which could be considerable in relative value when the IAM Rf value is as low as $3 \%$.

A significant difference in the ADPs of non-H atoms was observed between IAM and TAAM refinements. The $U_{\text {eq }}$ parameters from TAAM were found to be higher than those from IAM for non-H atoms [Fig. 3(a)]. This trend is opposite to what is observed in the X-ray structure refinement where the $U_{\text {eq }}$ values for non- $\mathrm{H}$ atoms were found to be lower in the case of X-ray TAAM refinement compared with X-ray IAM refinement (Jelsch et al., 1998; Jha et al., 2020). The advantage of multipolar TAAM refinement over IAM refinement has been established in the case of X-ray diffraction data on several occasions for atomic positions and ADPs (Volkov et al., 2004, 2007; Zarychta et al., 2007; Bąk et al., 2011, Jha et al., 2020) and more recently on electron diffraction data (Gruza et al., 2020).

The smaller values of $U_{\text {eq }}$ from IAM (average $U_{\text {eq }}=$ $0.0105 \AA^{2}$ ) compared with those from TAAM (average $U_{\text {eq }}=$ $0.0135 \AA^{2}$ ) correspond well to the differences in molecular ESPs computed from these two models. The ESP generated by the TAAM multipolar charged atoms model can be negative in some regions, contrary to the IAM ESP (Fig. 7). The TAAM ESP is notably shifted towards negative values locally in the covalent bond regions (Fig. 7). In real space, the TAAM potential appears more contracted than the IAM potential.

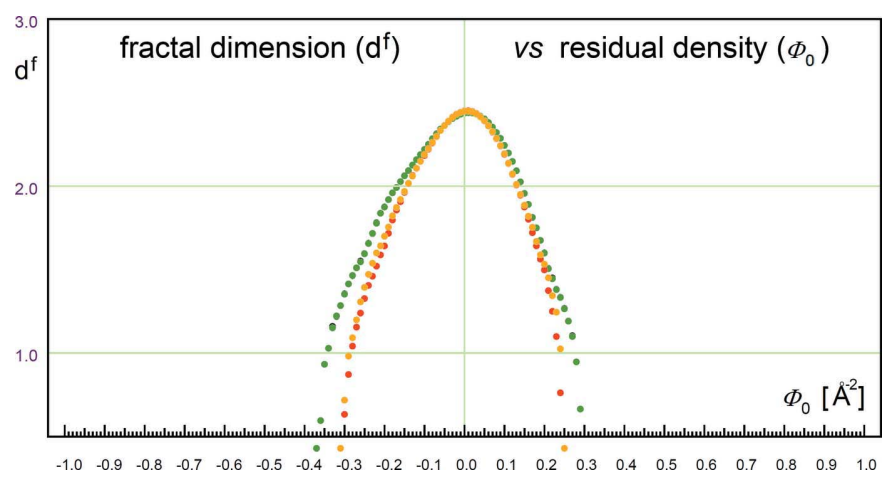

Figure 5

Comparison of fractal dimension for IAM with electron scattering factors from Peng (1999) (black) and IT (green), and for TAAM using the ELMAM2 (red) and UBDB (yellow) databanks. The fractal dimensions were obtained using the $j n k 2 R D A$ program (Henn \& Meindl, 2014).

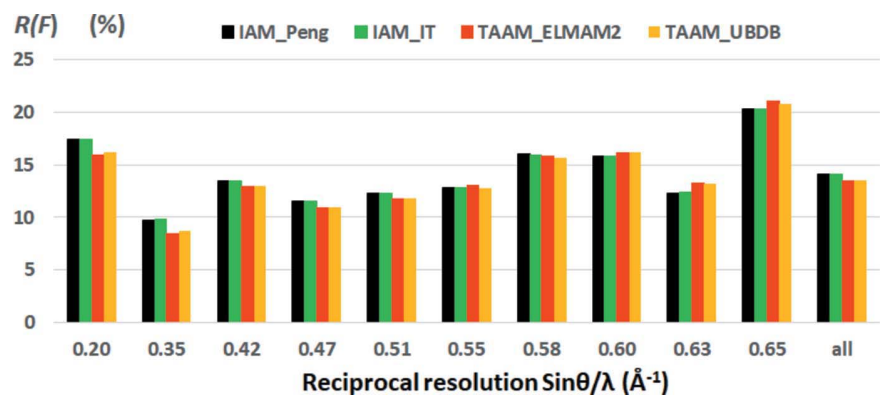

Figure 6

Reliability factor (Rf) (\%) as a function of reciprocal resolution $\left(\AA^{-1}\right)$ for MoPro refinements with IAM and TAAM.
When using the IAM model, the ESP is more diffused and, to compensate for this, the ADPs appear smaller to fit better the true molecular ESP. The bias in IAM potential is clearly visible

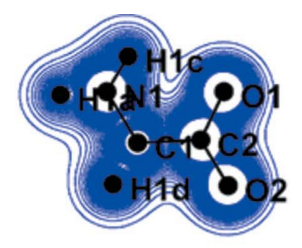

IAM

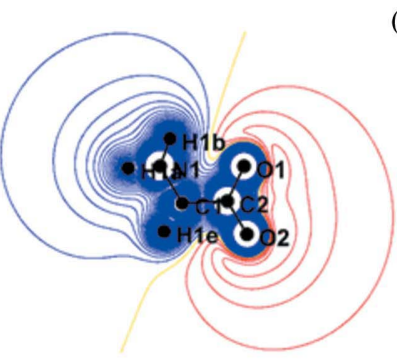

TAAM_ELMAM2

(b)

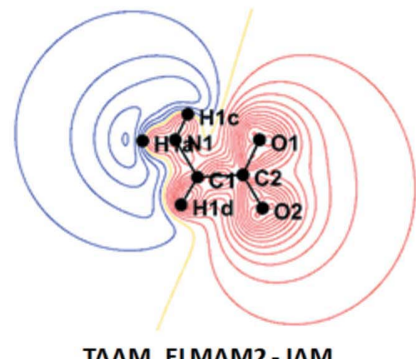

(d)

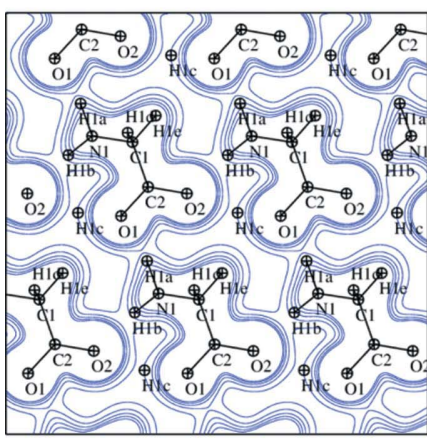

static ELMAM2

(f) (a)

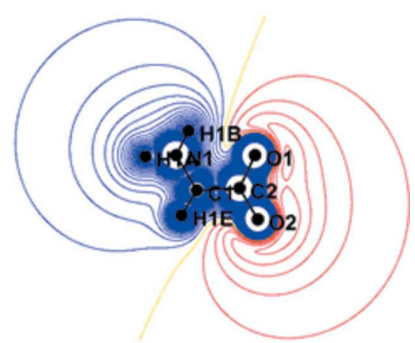

TAAM_UBDB

(c)

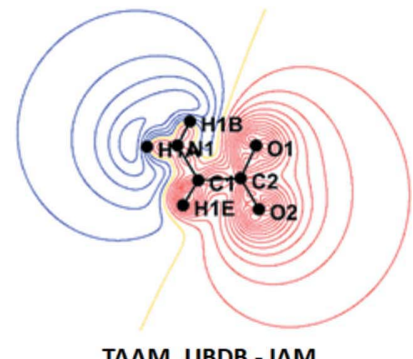

(e)

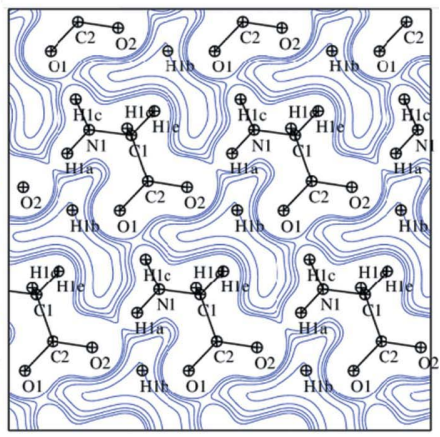

static IAM

(g)
Figure 7

Total static electrostatic potential $\left(\mathrm{e} \AA^{-1}\right.$ ) for an isolated $\beta$-glycine molecule obtained from various methods: $(a)$ IAM Peng, $(b)$ TAAM ELMAM2 and $(c)$ TAAM UBDB. $(d),(e)$ Deformation electrostatic potential. $(f)$ Static total ESP map, which approaches the periodic ESP generated by $19^{3}$ unit cells obtained by translations up to $[-9,9](\mathbf{a}, \mathbf{b}, \mathbf{c})$. The plane contains the $\mathrm{O} 2$ atom and is parallel to $(\mathbf{a}, \mathbf{c})$. ( $g$ ) Equivalent static periodic IAM ESP map. The properties were obtained directly from the multipolar models without passing through Fourier transformations. Contours are at 0.05 e $\AA^{-1}$ intervals (blue - positive and red - negative). The values of the deformation potential (TAAM-IAM) in the asymmetric unit range from $\Phi_{\min }=-0.66 \mathrm{e} \AA^{-1}$ to $\Phi_{\max }=+0.25 \mathrm{e} \AA^{-1}$ for ELMAM2 (d) and $\Phi_{\min }=-0.71$ e $\AA^{-1}$ to $\Phi_{\max }=+0.28$ e $\AA^{-1}$ for UBDB $(e)$. For the periodic TAAM ELMAM2 $(f)$ and IAM total $(g)$ ESP in the plotted plane, $\Phi_{\min }=+0.012$ and $+0.032 \mathrm{e}^{-1}$, respectively. 
in Fig. 7. The IAM potential is too positive in the covalent bonding and electron pair regions, and too small in the regions surrounding the polar electropositive $\mathrm{H}$ atoms. In addition, glycine is zwitterionic, but in the IAM ESP map there is no negative potential region surrounding the negatively charged carboxylate group. Regions of negative potential are clearly seen in the TAAM potential maps (Fig. 7). On top of that, the presence of dynamic scattering in the experimental data may influence the refined values of ADPs. Dynamic scattering lowers the intensities of low-order reflections and increases the intensities of high-order reflections on average (Clabbers et al., 2019). To compensate for this effect, ADPs in the kinematic model (either for IAM or for TAAM) may tend to be too small to fit better to experimental data containing dynamic effects. Nevertheless, in addition to observed changes in size, an improvement in the shapes of ellipsoids was observed in TAAM refinements compared with IAM refinements in MoPro (Figs. S3 and S4). This indicates that TAAM allows one to achieve a more physical structural model.

Quasi-periodic static total ESP maps were also computed in real space from the ELMAM2 model [Fig. 7(f)]. When the crystal environment is taken into account, the regions of negative potential tend to disappear for $\beta$-glycine, as the positive potential of neighbouring molecules is higher. The minimum TAAM_ELMAM2 ESP value in the plane shown in Fig. $7(f)$ is for instance $0.012 \mathrm{e}^{-1}$, which is however lower

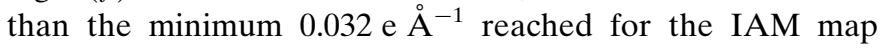
[Fig. 7(g)].

There was not much difference in the hydrogen $U_{\text {iso }}$ values found except in one case $(\mathrm{H} 1 B)$, where an unusually higher $U_{\text {iso }}$ value resulted in the case of TAAM refinement from ELMAM2 [Fig. 3(b)]. The non-H bond lengths were least affected by the method used and were found to be almost the same in the IAM and TAAM models [Fig. 3(c) and Table S3]. There were variations in the $\mathrm{H}-X$ bond lengths between IAM and TAAM refinements. TAAM $\mathrm{H}-X$ bond lengths were found to be slightly shorter than those from IAM. Previously, it was observed that IAM 3D ED refinements produce longer $\mathrm{H}-X$ bond lengths than TAAM, the latter being closer to reference neutron diffraction bond lengths (Gruza et al., 2020), which agrees with our observation.

\section{Conclusion}

Spherical and aspherical refinement on small-molecule electron diffraction data is now possible with the MoPro software with two different implementations of electron scattering factors. A statistical comparison of spherical IAM from SHELX and MoPro produced very similar results. No significant difference in ADPs and bond distances was found in IAM of both the non- $\mathrm{H}$ and $\mathrm{H}$ atoms from SHELX and MoPro refined structures. TAAM refinement in MoPro using various pseudoatom databanks can be readily performed with MoPro. TAAM refinement using ELMAM2 and UBDB pseudoatom databanks gave similar $\mathrm{Rf}$ values and the atomic positions were also found to be almost same. The major differences in statistics appeared between IAM and TAAM refinements. TAAM gives a better fit to the low-resolution region of $3 \mathrm{D}$ ED data (up to $\sin \theta / \lambda=0.5 \mathrm{~A}^{-1}$ ) compared with IAM. The Rf values from TAAM refinement were found to be lower than IAM globally at lower resolution, while at higher resolution the trends were not clear. Overall, a significant decrease in $\operatorname{Rf}(c a \sim 0.7 \%)$ and residuals $\left(c a 0.05-0.06 \AA^{-2}\right)$ in TAAM compared with IAM refinement indicated a better fit of TAAM to the diffraction data. A significant improvement in fractal dimension and lower $\Delta \Phi_{\mathrm{o}}$ of TAAM refinement compared with IAM also indicates the better fit of the structural model obtained from TAAM refinement.

The IAM description leads to a systematic error, as the ESP generated by a molecule is positive everywhere. Glycine is zwitterionic and its negative potential region around the carboxylate group is clearly seen in the TAAM potential maps, but it is not present in the IAM potential map. The negative potential disappears, however, in the total static periodic ESP map when the surrounding molecules in the crystal are taken into account for the ESP calculation. The positive potential in the approximated static periodic ESP map for TAAM is still significantly lower than that for IAM, with a distinct surface boundary for the carboxylate and ammonium moieties.

ADPs for non-H atoms are larger for TAAM than for IAM, following the trend previously observed for carbamazepine. The $\mathrm{H}$ atoms have similar $U_{\text {iso }}$ values. Contrarily to X-ray TAAM refinement on experimental data, the larger ADPs for non-H atoms in TAAM refinement may not indicate a better representation of thermal motion. Knowing that TAAM cannot correct the large measurement errors due to the dynamic scattering and in the absence of neutron diffraction data of $\beta$-glycine, the validation of the ADPs, especially for $\mathrm{H}$ atoms, is currently not possible. Nevertheless, the ELMAM2 and UBDB databank refinements gave similar ADPs. In addition, an improvement in the shapes of ellipsoids was observed in the TAAM refinement compared with the IAM refinement in MoPro. The non-H bond lengths remain the same irrespective of the model used, but major differences appear in the $\mathrm{H}-X$ bond lengths. The $\mathrm{H}-X$ bond lengths from IAM are comparatively large compared with those from the TAAM structures and with standard distances obtained from neutron diffraction (Allen \& Bruno, 2010). These results establish the applicability of MoPro in spherical IAM refinement of electron diffraction data of small molecules with a similar accuracy to other well known software like SHELX or OLEX2.

The multipolar electron density of small organic compounds can be transferred from a database such as UBDB or ELMAM2. The resulting multipolar model can be readily used for refinement against electron diffraction data with MoPro thanks to the Mott-Bethe transformation described in equation (1). In addition, MoPro facilitates the interpretation of properties obtained directly from the model, such as ESP, electrostatic interaction energies etc., which otherwise are not accessible currently in other programs. In the future, the improvement in experimental data such as higher completeness, higher redundancy, more constant volumes of a crystal being illuminated by a beam, less radiation damage and a 
lesser impact of dynamic scattering is expected with the use of better techniques and thinner crystals, which will further enhance the improvement obtained from TAAM compared with IAM. Furthermore, the methodology for refinement of 3D ED data of macromolecules will also be developed in MoPro.

\section{Funding information}

Support of this work by the National Centre of Science (Poland) through grant OPUS No. UMO-2017/27/B/ST4/ 02721 and by PL-Grid Infrastructure through grant PLGUBDB2020 is gratefully acknowledged.

\section{References}

Allen, F. H. \& Bruno, I. J. (2010). Acta Cryst. B66, 380-386.

Bąk, J. M., Domagała, S., Hübschle, C., Jelsch, C., Dittrich, B. \& Dominiak, P. M. (2011). Acta Cryst. A67, 141-153.

Beale, E. V., Waterman, D. G., Hecksel, C., van Rooyen, J., Gilchrist, J. B., Parkhurst, J. M., de Haas, F., Buijsse, B., Evans, G. \& Zhang, P. (2020). Front. Mol. Biosci. 7, 179.

Becker, P. \& Coppens, P. (1990). Acta Cryst. A46, 254-258.

Bibila Mayaya Bisseyou, Y., Bouhmaida, N., Guillot, B., Lecomte, C., Lugan, N., Ghermani, N. \& Jelsch, C. (2012). Acta Cryst. B68, 646660.

Blum, T. B., Housset, D., Clabbers, M. T. B., van Genderen, E., BaciaVerloop, M., Zander, U., McCarthy, A. A., Schoehn, G., Ling, W. L. \& Abrahams, J. P. (2021). Acta Cryst. D77, 75-85.

Boldyreva, E. V., Drebushchak, V. A., Drebushchak, T. N., Paukov, I. E., Kovalevskaya, Y. A. \& Shutova, E. S. (2003). J. Therm. Anal. Calorim. 73, 409-418.

Bouhmaida, N., Bonhomme, F., Guillot, B., Jelsch, C. \& Ghermani, N. E. (2009). Acta Cryst. B65, 363-374.

Brázda, P., Palatinus, L. \& Babor, M. (2019). Science, 364, 667-669.

Broadhurst, E. T., Xu, H., Clabbers, M. T. B., Lightowler, M., Nudelman, F., Zou, X. \& Parsons, S. (2020). IUCrJ, 7, 5-9.

Brock, C. P., Dunitz, J. D. \& Hirshfeld, F. L. (1991). Acta Cryst. B47, 789-797.

Brown, A., Long, F., Nicholls, R. A., Toots, J., Emsley, P. \& Murshudov, G. (2015). Acta Cryst. D71, 136-153.

Clabbers, M. T. B., van Genderen, E., Wan, W., Wiegers, E. L., Gruene, T. \& Abrahams, J. P. (2017). Acta Cryst. D73, 738-748.

Clabbers, M. T. B., Gruene, T., van Genderen, E. \& Abrahams, J. P. (2019). Acta Cryst. A75, 82-93.

Clementi, E. \& Roetti, C. (1974). At. Data Nucl. Data Tables, 14, 177478.

Cowley, J. M. \& Moodie, A. F. (1957). Acta Cryst. 10, 609-619.

Cruz, M. J. de la, Hattne, J., Shi, D., Seidler, P., Rodriguez, J., Reyes, F. E., Sawaya, M. R., Cascio, D., Weiss, S. C., Kim, S. K., Hinck, C. S., Hinck, A. P., Calero, G., Eisenberg, D. \& Gonen, T. (2017). Nat. Methods, 14, 399-402.

Dittrich, B., Hübschle, C. B., Pröpper, K., Dietrich, F., Stolper, T. \& Holstein, J. J. (2013). Acta Cryst. B69, 91-104.

Dittrich, B., Koritsánszky, T. \& Luger, P. (2004). Angew. Chem. Int. Ed. 43, 2718-2721.

Dolomanov, O. V., Bourhis, L. J., Gildea, R. J., Howard, J. A. K. \& Puschmann, H. (2009). J. Appl. Cryst. 42, 339-341.

Domagała, S., Fournier, B., Liebschner, D., Guillot, B. \& Jelsch, C. (2012). Acta Cryst. A68, 337-351.

Dominiak, P. M., Volkov, A., Li, X., Messerschmidt, M. \& Coppens, P. (2007). J. Chem. Theory Comput. 3, 232-247.

Gemmi, M., Mugnaioli, E., Gorelik, T. E., Kolb, U., Palatinus, L., Boullay, P., Hovmöller, S. \& Abrahams, J. P. (2019). ACS Cent. Sci. 5, 1315-1329.
Genderen, E. van, Clabbers, M. T. B., Das, P. P., Stewart, A., Nederlof, I., Barentsen, K. C., Portillo, Q., Pannu, N. S., Nicolopoulos, S., Gruene, T. \& Abrahams, J. P. (2016). Acta Cryst. A72, 236-242.

Gruene, T., Wennmacher, J. T. C., Zaubitzer, C., Holstein, J. J., Heidler, J., Fecteau-Lefebvre, A., De Carlo, S., Müller, E., Goldie, K. N., Regeni, I., Li, T., Santiso-Quinones, G., Steinfeld, G., Handschin, S., van Genderen, E., van Bokhoven, J. A., Clever, G. H. \& Pantelic, R. (2018). Angew. Chem. Int. Ed. 57, 16313-16317.

Gruza, B., Chodkiewicz, M. L., Krzeszczakowska, J. \& Dominiak, P. M. (2020). Acta Cryst. A76, 92-109.

Guillot, B., Jelsch, C., Podjarny, A. \& Lecomte, C. (2008). Acta Cryst. D64, 567-588.

Guillot, B., Viry, L., Guillot, R., Lecomte, C. \& Jelsch, C. (2001). J. Appl. Cryst. 34, 214-223.

Hansen, N. K. \& Coppens, P. (1978). Acta Cryst. A34, 909-921.

Hattne, J., Martynowycz, M. W., Penczek, P. A. \& Gonen, T. (2019). IUCrJ, 6, 921-926.

Henn, J. \& Meindl, K. (2014). Acta Cryst. A70, 499-513.

Jarzembska, K. N. \& Dominiak, P. M. (2012). Acta Cryst. A68, 139147.

Jelsch, C., Devi, R. N., Noll, B. C., Guillot, B., Samuel, I. \& Aubert, E. (2020). J. Mol. Struct. 1205, 127600.

Jelsch, C., Guillot, B., Lagoutte, A. \& Lecomte, C. (2005). J. Appl. Cryst. 38, 38-54.

Jelsch, C., Pichon-Pesme, V., Lecomte, C. \& Aubry, A. (1998). Acta Cryst. D54, 1306-1318.

Jha, K. K., Gruza, B., Kumar, P., Chodkiewicz, M. L. \& Dominiak, P. M. (2020). Acta Cryst. B76, 296-306.

Jones, C. G., Martynowycz, M. W., Hattne, J., Fulton, T. J., Stoltz, B. M., Rodriguez, J. A., Nelson, H. M. \& Gonen, T. (2018). ACS Cent. Sci. 4, 1587-1592.

Kolb, U., Mugnaioli, E. \& Gorelik, T. E. (2011). Cryst. Res. Technol. 46, 542-554.

Krysiak, Y., Barton, B., Marler, B., Neder, R. B. \& Kolb, U. (2018). Acta Cryst. A74, 93-101.

Kumar, P., Gruza, B., Bojarowski, S. A. \& Dominiak, P. M. (2019). Acta Cryst. A75, 398-408.

Meindl, K. \& Henn, J. (2008). Acta Cryst. A64, 404-418.

Mott, N. F. \& Massey, H. S. W. (1965). Theory of Atomic Collision, 3rd ed. Oxford, New York: Clarendon Press/Oxford University Press.

Mugnaioli, E., Lanza, A. E., Bortolozzi, G., Righi, L., Merlini, M., Cappello, V., Marini, L., Athanassiou, A. \& Gemmi, M. (2020). ACS Cent. Sci. 6, 1578-1586.

Nakashima, P. N. H. (2017). Struct. Chem. 28, 1319-1332.

Nakashima, P. N. H., Smith, A. E., Etheridge, J. \& Muddle, B. C. (2011). Science, 331, 1583-1586.

Nannenga, B. L. \& Gonen, T. (2019). Nat. Methods, 16, 369-379.

Nannenga, B. L., Shi, D., Leslie, A. G. W. \& Gonen, T. (2014). Nat. Methods, 11, 927-930.

Nassour, A., Domagala, S., Guillot, B., Leduc, T., Lecomte, C. \& Jelsch, C. (2017). Acta Cryst. B73, 610-625.

Niranjana Devi, R., Jelsch, C., Israel, S., Aubert, E., Anzline, C. \& Hosamani, A. A. (2017). Acta Cryst. B73, 10-22.

Palatinus, L., Petříček, V. \& Corrêa, C. A. (2015). Acta Cryst. A71, 235-244.

Peng, L.-M. (1999). Micron, 30, 625-648.

Perlovich, G. L., Hansen, L. K. \& Bauer-Brandl, A. (2001). J. Therm. Anal. Cal, 66, 699-715.

Petříček, V., Dušek, M. \& Palatinus, L. (2014). Z. Krist. Mater. 229, 345-352.

Polovinkin, V., Khakurel, K., Babiak, M., Angelov, B., Schneider, B., Dohnalek, J., Andreasson, J. \& Hajdu, J. (2020). J. Appl. Cryst. 53, 1416-1424.

Prince, E. (2006). Editor. International Tables for X-ray Crystallography, Vol. C, Mathematical, Physical and Chemical Tables, 1st online ed., ch. 4.3, pp. 259-429. Chester: International Union of Crystallography. 
Sawaya, M. R., Rodriguez, J., Cascio, D., Collazo, M. J., Shi, D., Reyes, F. E., Hattne, J., Gonen, T. \& Eisenberg, D. S. (2016). Proc. Natl Acad. Sci. USA, 113, 11232-11236.

Sheldrick, G. M. (2015). Acta Cryst. C71, 3-8.

Shi, D., Nannenga, B. L., Iadanza, M. G. \& Gonen, T. (2013). eLife, 2 , e01345.

Thorn, A., Dittrich, B. \& Sheldrick, G. M. (2012). Acta Cryst. A68, 448-451.

Volkov, A., Li, X., Koritsanszky, T. \& Coppens, P. (2004). J. Phys. Chem. A, 108, 4283-4300.

Volkov, A., Messerschmidt, M. \& Coppens, P. (2007). Acta Cryst. D63, 160-170.

Wang, B., Zou, X. \& Smeets, S. (2019). IUCrJ, 6, 854-867.

Watkin, D. (2008). J. Appl. Cryst. 41, 491-522.

Xu, H. \& Zou, X. (2019). Science, 364, 632-633.
Yonekura, K., Kato, K., Ogasawara, M., Tomita, M. \& Toyoshima, C. (2015). Proc. Natl Acad. Sci. USA, 112, 3368-3373.

Yonekura, K. \& Maki-Yonekura, S. (2016). J. Appl. Cryst. 49, 15171523.

Yonekura, K., Matsuoka, R., Yamashita, Y., Yamane, T., Ikeguchi, M., Kidera, A. \& Maki-Yonekura, S. (2018). IUCrJ, 5, 348-353.

Zarychta, B., Pichon-Pesme, V., Guillot, B., Lecomte, C. \& Jelsch, C. (2007). Acta Cryst. A63, 108-125.

Zheng, J.-C., Wu, L. \& Zhu, Y. (2009). J. Appl. Cryst. 42, 1043-1053. Zheng, J.-C., Zhu, Y., Wu, L. \& Davenport, J. W. (2005). J. Appl. Cryst. 38, 648-656.

Zhong, S., Dadarlat, V. M., Glaeser, R. M., Head-Gordon, T. \& Downing, K. H. (2002). Acta Cryst. A58, 162-170.

Zuo, J. M., Kim, M., O'Keeffe, M. \& Spence, J. C. H. (1999). Nature, 401, 49-52. 